\title{
Anecdote Increases Patient Willingness to Take a Biologic Medication for Psoriasis
}

\author{
Matthew C. Johnson, MD; Elias Oussedik, MD; William Wei-Ting Huang, MD, MPH; \\ Lara K. Kammrath, PhD; Steven R. Feldman, MD, PhD
}

\section{PRACTICE POINTS}

- Patients often are apprehensive to start biologic medications for their psoriasis.

- Clinical trial evidence of a biologic medication's efficacy and safety as well as anecdotes of patient experiences appear to be important factors for patients when considering taking a medication.

- The use of an anecdote-alone or in combination with clinical trial evidence-to help patients overcome fears of starting a biologic medication for their psoriasis may be an effective way to improve patients' willingness to take treatment. or in combination with clinical trial evidence-may be an effective way to improve patients' willingness to take a biologic medication for psoriasis.

Cutis. 2021;108(suppl 2):20-24, E7.

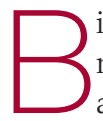
iologic medications are highly effective in treating moderate to severe psoriasis, yet many patients are apprehensive about taking a biologic medication for a variety of reasons, such as hearing negative information about the drug from friends or family, being nervous about injection, or seeing the drug or its side effects negatively portrayed in the media. ${ }^{1-3}$ Because biologic medications are costly, many patients may fear needing to discontinue use of the medication owing to lack of affordability, which may result in subsequent rebound of psoriasis. Because patients' fear of a drug is inherently subjective, it can be modified with appropriate reassurance and presentation of evidence. By understanding what information increases patients' confidence in their willingness to take a biologic medication, patients may be more willing to initiate use of the drug and improve treatment outcomes.

There are mixed findings about whether statistical evidence or an anecdote is more effective in persuasion..$^{4-6}$ The specific context in which the persuasion takes place

Drs. Johnson, Oussedik, Huang, and Feldman are from the Center for Dermatology Research, Department of Dermatology, Wake Forest School of Medicine, Winston-Salem, North Carolina. Dr. Feldman also is from the Departments of Pathology and Social Sciences \& Health Policy. Dr. Oussedik also is from the Faculty of Medicine, McGill University, Montreal, Quebec, Canada. Dr. Kammrath is from the Department of Psychology, Wake Forest University, Winston-Salem.

Drs Johnson, Oussedik, Huang, and Kammrath report no conflict of interest. Dr. Feldman has received research, speaking, and/or consulting support from the following companies: AbbVie; Janssen Pharmaceuticals; National Biological Corporation; the National Psoriasis Foundation; Novartis; Pfizer Inc; Samsung Group; and Sun Pharmaceutical Industries Ltd. He also is part owner of Causa Research, a company dedicated to enhancing patients' adherence to treatment.

The eTable is available in the Appendix online at www.mdedge.com/dermatology.

Correspondence: Steven R. Feldman, MD, PhD, Department of Dermatology, Wake Forest School of Medicine, Medical Center Blvd, Winston-Salem, NC 27157-1071 (sfeldman@wakehealth.edu).

doi:10.12788/cutis.0320 
may be important in determining which method is superior. In most nonthreatening situations, people appear to be more easily persuaded by statistical evidence rather than an anecdote. However, in circumstances where emotional engagement is high, such as regarding one's own health, an anecdote tends to be more persuasive compared to statistical evidence. ${ }^{7}$ The purpose of this study was to evaluate patients' willingness to take a biologic medication for the management of their psoriasis if presented with either clinical trial evidence of the agent's efficacy and safety, an anecdote of a single patient's positive experience, or both.

\section{Methods}

Patient Inclusion Criteria-Following Wake Forest School of Medicine institutional review board approval, a prospective parallel-arm survey study was performed on eligible patients 18 years or older with a self-reported diagnosis of psoriasis. Patients were required to have a working knowledge of English and not have been previously prescribed a biologic medication for their psoriasis. If patients did not meet inclusion criteria after answering the survey eligibility screening questions, then they were unable to complete the remainder of the survey and were excluded from the analysis.

Survey Administration-A total of 222 patients were recruited through Amazon Mechanical Turk, an online crowdsourcing platform. (Amazon Mechanical Turk is a validated tool in conducting research in psychology and other social sciences and is considered as diverse as and perhaps more representative than traditional samples. ${ }^{8,9}$ ) Patients received a fact sheet and were taken to the survey hosted on Qualtrics, a secure web-based survey software that supports data collection for research studies. Amazon Mechanical Turk requires some amount of compensation to patients; therefore, recruited patients were compensated $\$ 0.03$.

Statistical Analysis-Patients were randomized using SPSS Statistics version 23.0 (IBM) in a 1:1 ratio to assess how willing they would be to take a biologic medication for their psoriasis if presented with one of the following: (1) a control that queried patients about their willingness to take treatment without having been informed on its efficacy or safety, (2) clinical trial evidence of the agent's efficacy and safety, (3) an anecdote of a single patient's positive experience, or (4) both clinical trial evidence of the agent's efficacy and safety and an anecdote of a single patient's positive experience (Table 1). Demographic information including sex, age, ethnicity, and education level was collected, in addition to other baseline characteristics such as having friends or family with a history of psoriasis, history of participation in a clinical trial with use of an experimental drug, and the number of years since clinical diagnosis of psoriasis.

Outcome measures were recorded as patients' responses regarding their willingness to take a biologic medication on a 10 -point Likert scale (1=not willing; $10=$ completely

\section{TABLE 1. Survey questions influencing psoriasis treatment ${ }^{2}$}

Group 1 (control)

How willing would you be to take a doctor-recommended treatment for your psoriasis while not having been informed on its efficacy or safety profile?

\section{Group 2 (clinical trial evidence)}

How willing would you be to take a treatment that has a $70 \%$ chance of nearly completely clearing your psoriasis, that has a $90 \%$ chance of very good improvement, and that was very safe in a research study of over 3000 patients, with only about a 1 in 300 chance of developing new or worsening inflammatory bowel disease?

\section{Group 3 (anecdote)}

How willing would you be to take a treatment if, in the doctor's experience, another patient with psoriasis very much like yours had near-complete clearing of their psoriasis and had no side effects?

\section{Group 4 (clinical trial evidence and anecdote)}

How willing would you be to take a treatment that has a $70 \%$ chance of nearly completely clearing your psoriasis, that has a $90 \%$ chance of very good improvement, and that was very safe in a research study of over 3000 patients, with only about a 1 in 300 chance of developing new or worsening inflammatory bowel disease; and if, in the doctor's experience, another patient with psoriasis very much like yours had near-complete clearing of their psoriasis and had no side effects?

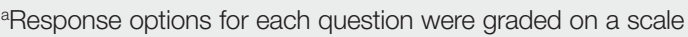
of 1 (not willing) to 10 (completely willing).

willing). Scores were treated as ordinal data and evaluated using the Kruskal-Wallis test followed by the Dunn test. Descriptive statistics were tabulated on all variables. Baseline characteristics were analyzed using a 2-tailed, unpaired $t$ test for continuous variables and the $\chi^{2}$ and Fisher exact tests for categorical variables. Ordinal linear regression analysis was performed to determine whether reported willingness to take a biologic medication was related to patients' demographics, including age, sex, having family or friends with a history of psoriasis, history of participation in a clinical trial with use of an experimental drug, and the number of years since clinical diagnosis of psoriasis. Answers on the ordinal scale were binarized. The data were analyzed with SPSS Statistics version 23.0.

\section{Results}

There were no statistically significant differences among the baseline characteristics of the 4 information assignment groups (Table 2). Patients in the control group not given either clinical trial evidence of a biologic medication's efficacy and safety or anecdote of a single patient's positive experience had the lowest reported willingness to take treatment (median, 4.0)(Figure). Patients in the group given 
TABLE 2. Summary of baseline characteristics $(\mathrm{N}=222)$

\begin{tabular}{|c|c|c|c|c|}
\hline Variable & $\begin{array}{l}\text { Control group } \\
(n=52)\end{array}$ & $\begin{array}{l}\text { Clinical trial } \\
\text { evidence group } \\
(n=47)\end{array}$ & $\begin{array}{l}\text { Anecdote group } \\
(n=58)\end{array}$ & $\begin{array}{l}\text { Clinical trial } \\
\text { evidence+ } \\
\text { anecdote group } \\
(n=65)\end{array}$ \\
\hline Mean age (SD), y & $33.6(10.3)$ & $38.0(12.8)$ & $35.2(12.2)$ & $35.9(12.0)$ \\
\hline Male sex, n (\%) & $27(51.9)$ & $19(40.4)$ & $29(50.0)$ & $29(44.6)$ \\
\hline \multicolumn{5}{|l|}{ Ethnicity, n (\%) } \\
\hline American Indian or Alaska Native & $5(9.6)$ & $4(8.5)$ & $5(8.6)$ & $1(1.5)$ \\
\hline Asian & $18(34.6)$ & $13(27.7)$ & $18(31.0)$ & $26(40.0)$ \\
\hline Black or African American & $3(5.8)$ & $3(6.4)$ & $2(3.4)$ & 0 \\
\hline White & $24(46.2)$ & $23(48.9)$ & $30(51.7)$ & $31(47.7)$ \\
\hline Hispanic or Latino & $2(3.8)$ & $1(2.1)$ & $1(1.7)$ & $4(6.2)$ \\
\hline Native Hawaiian or Pacific Islander & 0 & $1(2.1)$ & $1(1.7)$ & $1(1.5)$ \\
\hline Other & 0 & $2(4.3)$ & $1(1.7)$ & $2(3.1)$ \\
\hline \multicolumn{5}{|l|}{ Education level, n (\%) } \\
\hline Advanced graduate work or $\mathrm{PhD}$ & $1(1.9)$ & 0 & 0 & $1(1.5)$ \\
\hline Associate's degree & $4(7.7)$ & $8(17.0)$ & $6(10.30$ & $10(15.4)$ \\
\hline Bachelor's degree & $25(48.1)$ & $14(29.8)$ & $25(43.1)$ & $25(38.5)$ \\
\hline Did not complete high school & $0(0)$ & $1(2.1)$ & $1(1.7)$ & $0(0)$ \\
\hline High school diploma/GED & 4( & $3(6.4)$ & $4(6.9)$ & $6(9.2)$ \\
\hline Master's degree & & $6(12.8)$ & $9(15.5)$ & $11(16.9)$ \\
\hline Not sure & $0(0)$ & $1(2.1)$ & $0(0)$ & $0(0)$ \\
\hline Some college & $10(19.2)$ & $14(29.8)$ & $13(22.4)$ & $12(18.5)$ \\
\hline \multicolumn{5}{|c|}{ History of participation in a clinical trial with use of an experimental drug, n (\%) } \\
\hline Yes & $23(44.2)$ & 15 (31.9) & $17(29.3)$ & $21(32.3)$ \\
\hline No & $29(55.8)$ & $32(68.1)$ & $41(70.7)$ & $44(67.7)$ \\
\hline \multicolumn{5}{|l|}{ Family or friends with psoriasis, $\mathrm{n}(\%)$} \\
\hline Yes & $20(38.5)$ & $19(40.4)$ & $23(39.7)$ & $22(33.8)$ \\
\hline No & $32(61.5)$ & 28 (59.6) & $35(60.3)$ & $43(66.2)$ \\
\hline Mean psoriasis duration (SD), y & $9.0(10.9)$ & $9.1(8.3)$ & $10.1(9.0)$ & $10.7(10.3)$ \\
\hline
\end{tabular}

Abbreviation: GED, General Education Development. 


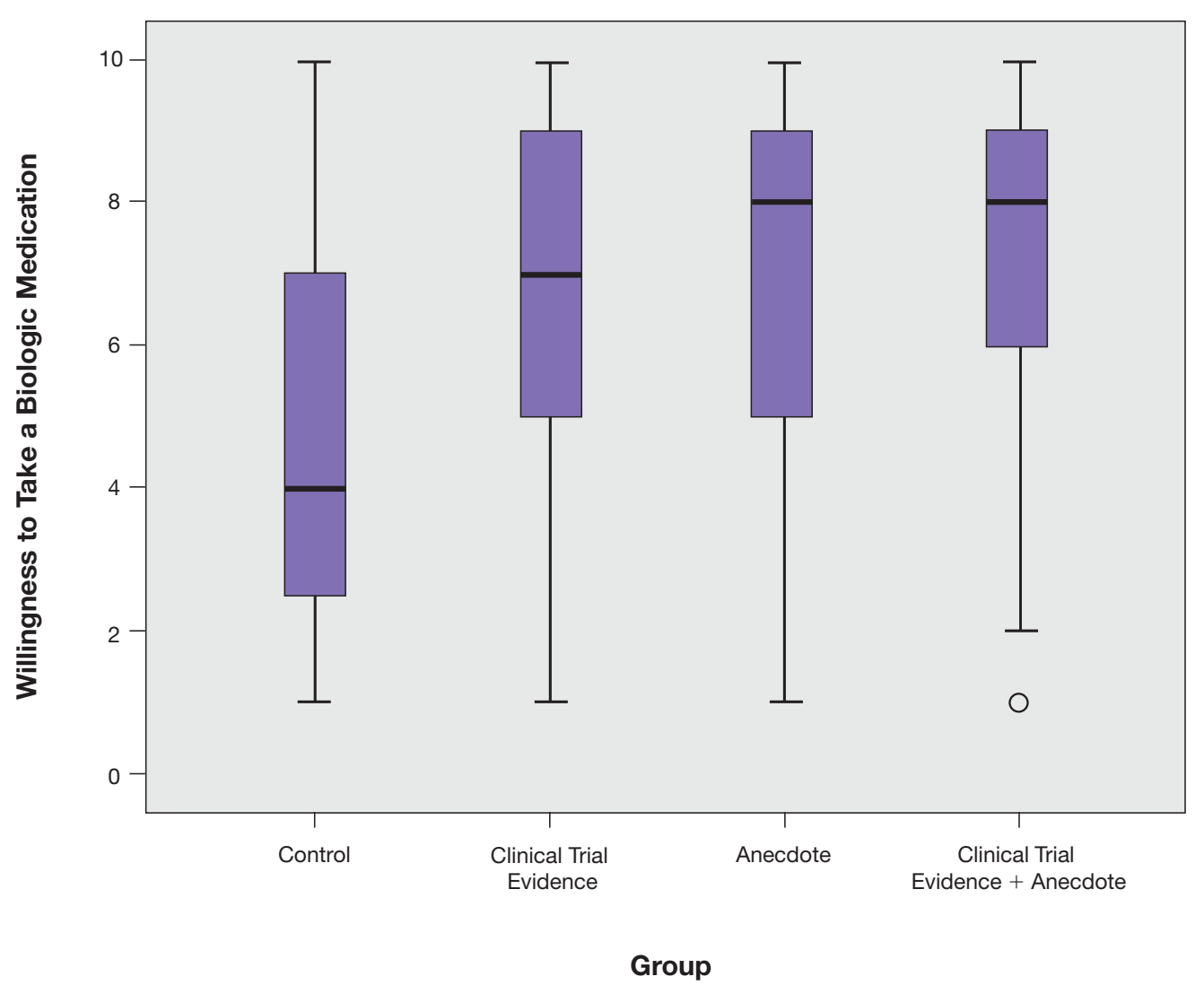

Willingness to take a biologic medication for the management of psoriasis in patients from 4 cohorts $(\mathrm{N}=222)$ graded on a 10-point Likert scale ( $1=$ not willing; $10=$ completely willing). The horizontal bar inside the boxes indicates the median, and the lower and upper ends of the boxes are the first and third quartiles. The whiskers extend to represent the lowest and highest observations for each cohort within 1.5 times the interquartile range (1.5IQR) below the first quartile and 1.5IQR above the third quartile. The small circle represents an outlier, as this value is smaller than $1.5 \mathrm{IQR}$ minus the first quartile.

clinical trial evidence had an intermediate level of reported willingness to take treatment (median, 7.0). Patients in the groups given an anecdote or clinical trial evidence and anecdote had the highest reported willingness to take treatment (median, 8.0). Patients presented with an anecdote or clinical trial evidence and anecdote were significantly more likely to report willingness to take treatment than those not presented with either clinical trial evidence or anecdote $(P<.001)$. Although patients presented with an anecdote or clinical trial evidence and anecdote were more likely to take a biologic medication than those presented with only clinical trial evidence, the difference between these groups was not significant $(P=.75)$.

Based on regression analysis, age, sex, and having friends or family with a history of psoriasis were not significantly associated with patients' responses (eTable). The number of years since clinical diagnosis of psoriasis $(P=.034)$ and history of participation in a clinical trial with use of an experimental drug $(P=.018)$ were significantly associated with the willingness of patients presented with an anecdote to take a biologic medication.

\section{Comment}

Anecdotal Reassurance-The presentation of clinical trial and/or anecdotal evidence had a strong effect on patients' willingness to take a biologic medication for their psoriasis. Human perception of a treatment is inherently subjective, and such perceptions can be modified with appropriate reassurance and presentation of evidence. ${ }^{1}$ Across the population we studied, presenting a brief anecdote of a single patient's positive experience is a quick and efficient means-and as or more effective as giving details on efficacy and safety-to help patients decide to take a treatment for their psoriasis.

Anecdotal reassurance is powerful. Both health care providers and patients have a natural tendency to focus on anecdotal experiences rather than statistical reasoning when making treatment decisions. ${ }^{10-12}$ Although negative anecdotal experiences may make patients unwilling to take a medication (or may make them overly desirous of an inappropriate treatment), clinicians can harness this psychological phenomenon to both increase patient willingness to take potentially beneficial treatments or to 
deter them from engaging in activities that can be harmful to their health, such as tanning and smoking.

Psoriasis Duration and Willingness to Take a Biologic Medication-In general, patient demographics did not appear to have an association with reported willingness to take a biologic medication for psoriasis. However, the number of years since clinical diagnosis of psoriasis had an effect on willingness to take a biologic medication, with patients with a longer personal history of psoriasis showing a higher willingness to take a treatment after being presented with an anecdote than patients with a shorter personal history of psoriasis. We can only speculate on the reasons why. Patients with a longer personal history of psoriasis may have tried and failed more treatments and therefore have a distrust in the validity of clinical trial evidence. These patients may feel their psoriasis is different than that of other clinical trial participants and thus may be more willing to rely on the success stories of individual patients.

Prior participation in a clinical trial with use of an experimental drug was associated with a lower willingness to choose treatment after being presented with anecdotal reassurance. This finding may be attributable to these patients understanding the subjective nature of anecdotes and preferring more objective information in the form of randomized clinical trials in making treatment decisions. Overall, the presentation of evidence about the efficacy and safety of biologic medications in the treatment of psoriasis has a greater impact on patient decision-making than patients' age, sex, and having friends or family with a history of psoriasis.

Limitations-Limitations of the study were typical of survey-based research. With closed-ended questions, patients were not able to explain their responses. In addition, hypothetical informational statements of a biologic's efficacy and safety may not always imitate clinical reality. However, we believe the study is valid in exploring the power of an anecdote in influencing patients' willingness to take biologic medications for psoriasis. Furthermore, educational level and ethnicity were excluded from the ordinal regression analysis because the assumption of parallel lines was not met.

Ethics Behind an Anecdote-An important consideration is the ethical implications of sharing an anecdote to guide patients' perceptions of treatment and behavior. Although clinicians rely heavily on the available data to determine the best course of treatment, providing patients with comprehensive information on all risks and benefits is rarely, if ever, feasible. Moreover, even objective clinical data will inevitably be subjectively interpreted by patients. For example, describing a medication side effect as occurring in 1 in 100 patients may discourage patients from pursuing treatment, whereas describing that risk as not occurring in 99 in 100 patients may encourage patients, despite these 2 choices being mathematically identical. ${ }^{13}$ Because the subjective interpretation of data is inevitable, presenting patients with subjective information in the form of an anecdote to help them overcome fears of starting treatment and achieve their desired clinical outcomes may be one of the appropriate approaches to present what is objectively the best option, particularly if the anecdote is representative of the expected treatment response. Clinicians can harness this understanding of human psychology to better educate patients about their treatment options while fulfilling their ethical duty to act in their patients' best interest.

\section{Conclusion}

Using an anecdote to help patients overcome fears of starting a biologic medication may be appropriate if the anecdote is reasonably representative of an expected treatment outcome. Patients should have an accurate understanding of the common risks and benefits of a medication for purposes of shared decision-making.

\section{REFERENCES}

1. Oussedik E, Cardwell LA, Patel NU, et al. An anchoring-based intervention to increase patient willingness to use injectable medication in psoriasis. JAMA Dermatol. 2017;153:932-934. doi:10.1001/ jamadermatol.2017.1271

2. Brown KK, Rehmus WE, Kimball AB. Determining the relative importance of patient motivations for nonadherence to topical corticosteroid therapy in psoriasis. J Am Acad Dermatol. 2006;55:607-613. doi:10.1016/j. jaad.2005.12.021

3. Im H, Huh J. Does health information in mass media help or hurt patients? Investigation of potential negative influence of mass media health information on patients' beliefs and medication regimen adherence. $J$ Health Commun. 2017;22:214-222. doi:10.1080/10810730.2016.1261970

4. Hornikx J. A review of experimental research on the relative persuasiveness of anecdotal, statistical, causal, and expert evidence. Studies Commun Sci. 2005;5:205-216.

5. Allen M, Preiss RW. Comparing the persuasiveness of narrative and statistical evidence using meta-analysis. Int J Phytoremediation Commun Res Rep. 1997;14:125-131. doi:10.1080/08824099709388654

6. Shen F, Sheer VC, Li R. Impact of narratives on persuasion in health communication: a meta-analysis. J Advert. 2015;44:105-113. doi:10.1080 /00913367.2015.1018467

7. Freling TH, Yang Z, Saini R, et al. When poignant stories outweigh cold hard facts: a meta-analysis of the anecdotal bias. Organ Behav Hum Decis Process. 2020;160:51-67. doi:10.1016/j.obhdp.2020.01.006

8. Buhrmester M, Kwang T, Gosling SD. Amazon's Mechanical Turk. Perspect Psychol Sci. 2011;6:3-5. doi:10.1177/1745691610393980

9. Berry K, Butt M, Kirby JS. Influence of information framing on patient decisions to treat actinic keratosis. JAMA Dermatol. 2017;153:421-426. doi:10.1001/jamadermatol.2016.5245

10. Landon BE, Reschovsky J, Reed M, et al. Personal, organizational, and market level influences on physicians' practice patterns: results of a national survey of primary care physicians. Med Care. 2001;39:889-905. doi:10.1097/00005650-200108000-00014

11. Borgida E, Nisbett RE. The differential impact of abstract vs. concrete information on decisions. J Appl Soc Psychol. 1977;7:258-271. doi:10.1111/j.1559-1816.1977.tb00750.x

12. Fagerlin A, Wang C, Ubel PA. Reducing the influence of anecdotal reasoning on people's health care decisions: is a picture worth a thousand statistics? Med Decis Making. 2005;25:398-405. doi:10.1177/0272989X05278931

13. Gurm HS, Litaker DG. Framing procedural risks to patients: Is $99 \%$ safe the same as a risk of 1 in 100? Acad Med. 2000;75:840-842. doi:10.1097/00001888-200008000-00018 


\section{APPENDIX}

\section{eTABLE. Ordinal Regression Analysis in Predicting Patient Willingness to Take a Biologic Medication for the Management of Psoriasis}

\begin{tabular}{|c|c|c|}
\hline Characteristic & $\mathrm{OR}^{\mathrm{a}}(95 \% \mathrm{Cl})$ & $P$ value \\
\hline \multicolumn{3}{|l|}{ Clinical trial evidence group } \\
\hline Age & $1.02(0.97-1.06)$ & .473 \\
\hline Sex & $0.43(0.14-1.30)$ & .133 \\
\hline History of participation in a clinical trial with use of an experimental drug ${ }^{b}$ & $0.42(0.13-1.39)$ & 157 \\
\hline Psoriasis duration ${ }^{c}$ & $0.99(0.93-1.05)$ & 5 \\
\hline Family or friends with psoriasis & $0.58(0.19-1.78)$ & 337 \\
\hline \multicolumn{3}{|l|}{ Anecdote group } \\
\hline Age & $1.00(0.96-1.04)$ & .981 \\
\hline Sex & $1.12(0.43-2.93)$ & .817 \\
\hline History of participation in a clinical trial with use of an experimental drug ${ }^{b}$ & $0.26(0.09-0.79)$ & .018 \\
\hline Psoriasis duration ${ }^{c}$ & $1.07(1.01-1.13)$ & .034 \\
\hline Family or friends with psoriasis & $0.78(0.30-2.03)$ & .614 \\
\hline \multicolumn{3}{|l|}{ Clinical trial evidence and anecdote group } \\
\hline Age & $1.01(0.97-1.04)$ & .753 \\
\hline Sex & $1.78(0.70-4.52)$ & .224 \\
\hline History of participation in a clinical trial with use of an experimental drug ${ }^{b}$ & $1.19(0.43-3.35)$ & .736 \\
\hline Psoriasis duration $^{c}$ & $1.02(0.97-1.06)$ & .496 \\
\hline Family or friends with psori & $0.72(0.28-1.88)$ & .505 \\
\hline
\end{tabular}

Abbreviation: OR, odds ratio.

${ }^{a} \mathrm{An}$ OR greater than 1 represents a positive association, while an OR less than 1 represents a negative association.

${ }^{\mathrm{b}}$ For every increasing year.

'For a positive report compared with no history of characteristic. 